This item was submitted to Loughborough's Research Repository by the author.

Items in Figshare are protected by copyright, with all rights reserved, unless otherwise indicated.

\title{
Predictor-based disturbance rejection control for sampled systems with input delay
}

PLEASE CITE THE PUBLISHED VERSION

https://doi.org/10.1109/TCST.2017.2781651

PUBLISHER

(C) IEEE

VERSION

AM (Accepted Manuscript)

LICENCE

CC BY-NC-ND 4.0

\section{REPOSITORY RECORD}

Liu, Tao, Shoulin Hao, Dewei Li, Wen-Hua Chen, and Qing-Guo Wang. 2017. "Predictor-based Disturbance Rejection Control for Sampled Systems with Input Delay". figshare. https://hdl.handle.net/2134/27717. 


\title{
Predictor-Based Disturbance Rejection Control for Sampled Systems with Input Delay
}

\author{
Tao Liu, Senior Member, IEEE, Shoulin Hao, Dewei Li, Wen-Hua Chen, Senior Member, IEEE, and \\ Qing-Guo Wang, Senior Member, IEEE
}

\begin{abstract}
To cope with input delay involved with many industrial system operations, a predictor-based disturbance rejection control (PDRC) scheme is proposed in discrete-time domain for sampling implementation, which can be generally applied for open-loop stable, integrating and unstable processes. An extended state observer (ESO) is introduced to estimate not only the deterministic system state but also the generalized disturbance composed of system uncertainties and external disturbance, based on a filtered Smith predictor to estimate the delay-free output response. Correspondingly, by specifying the desired poles of ESO and the closed-loop control system, the ESO gain vector and the PDRC controller are analytically derived. A notable merit is that there is a single tuning parameter in the proposed ESO, output predictor, and the PDRC controller, which can be monotonically tuned to achieve a good trade-off between the prediction (or control) performance and its robustness. A sufficient robust stability condition of the closed-loop system is established in terms of linear matrix inequality (LMI). An illustrative example from the literature along with an application to the temperature control system for a crystallization reactor is used to demonstrate the effectiveness and advantage of the proposed control method.
\end{abstract}

Index Terms-Sampled control systems, input delay, disturbance rejection control, dead-time compensator, extended state observer, robust stability.

\section{INTRODUCTION}

$\mathrm{T}$ ME delay is generally involved with industrial process operations, due to mass transportation, energy exchange, signal processing and execution etc. $[1,2]$, which may provoke performance deterioration even instability of the control system, in particular for the presence of input delay, or equivalently, delayed output response [3]. Hence, control of industrial systems with time delay, especially for input delay, has attracted considerable attentions in the recent years [4-9]. It has been well known that the conventional PID controller could

This work is supported in part by the NSF China Grants 61633006 and 61473054, and the National Thousand Talents Program of China.

Tao Liu and Shoulin Hao are with the Institute of Advanced Control Technology, Dalian University of Technology, Dalian, 116024, China (e-mail: liurouter@ieee.org; haoshoulin@gmail.com).

Dewei $\mathrm{Li}$ is with the Department of Automation, Shanghai Jiaotong University, Shanghai, China (e-mail: dwli@sjtu.edu.cn)

Wen-Hua Chen is with Department of Aeronautical and Automotive Engineering, Loughborough University, Loughborough, LE11 3TU, U.K. (e-mail: w.chen@1boro.ac.uk).

Qing-Guo Wang is with the Institute for Intelligent Systems, The University of Johannesburg, Johannesburg, 524, South Africa (e-mail: wangq@uj.ac.za). only be capable of controlling linear systems free of delay or having small time delay [10], inevitably resulting in poor performance in the presence of apparent time delay. Inspired by the Smith predictor (SP) [11] that aims at eliminating the delay from the characteristic equation for the closed-loop system transfer function, different dead-time compensators (DTCs) had been developed to facilitate control design for time delay systems [12]. In fact, the classical SP or the similar internal model control (IMC) scheme can only be used for stable processes with time delay due to the internal stability issue [13]. Modified SP or IMC designs have been proposed in the literature (e.g., $[4,5,14-18])$ for application to integrating and unstable processes with time delay. It was demonstrated that the filtered SP (FSP) [4, 5], which gives a delay-free output prediction, could be generally used for open-loop stable, integrating and unstable processes with time delay. In contrast, another dead-time compensator called generalized predictor (GP) was proposed in discrete-time domain $[6,7]$ for general application to stable, integrating, and unstable processes with time delay. However, no disturbance was considered in these references for designing the predictor, besides the output disturbance as commonly assumed in the IMC-based predictor [13]. It has been recognized that the design of a specific disturbance observer could estimate the dynamics of disturbance entering into the process not only from the output but also from the input, thus facilitating improving disturbance rejection control (DRC) performance $[19,20]$. It should be noted that the developed disturbance observer designs were mostly devoted to linear or nonlinear systems free of time delay or having small time delay that may be linearly approximated.

To counteract load disturbance and plant uncertainties, the active DRC (ADRC) strategy was developed which could lead to obvious performance improvement when compared to traditional controllers like PID [21], and therefore, has attracted increasing attention in the recent years [22-25]. The key idea of ADRC lies with treating external disturbance and unmodeled system dynamics as "a generalized disturbance", which is estimated by using a modified state observer, such that the feedback control could be specifically designed to eliminate the disturbance effect. However, the developed ADRC methods were mainly focused on delay-free systems. For time-delay systems, it was proposed [24] to design a modified ADRC to tackle the time delay, such that good disturbance rejection could be obtained while maintaining the system stability compared with the standard ADRC. Nevertheless, this method could not deal with relatively larger time delay. Another 
predictive ADRC was developed [25] to compensate for the input delay for disturbance rejection, which could obtain further improved performance, but is only applicable for stable processes with time delay.

In this paper, a predictor-based DRC (PDRC) scheme is proposed for industrial sampled systems with input delay, which can be generally applied for open-loop stable, integrating and unstable processes. Based on using an FSP for delay-free output prediction, an extended state observer (ESO) is introduced to estimate not only the deterministic system state but also system uncertainties and external disturbance. By specifying the desired poles of ESO and the closed-loop control system, the ESO gain vector and the PDRC controller are analytically derived, respectively. Meanwhile, a sufficient robust stability condition of the control system is established in terms of linear matrix inequality (LMI).

For clarity, the paper is organized as follows. In Section II, problem statement and preliminaries are presented. The proposed PDRC scheme is detailed in Section III. Robust stability of the closed-loop system is analyzed in Section IV. An illustrative example is shown in Section $\mathrm{V}$, followed by temperature control tests for a crystallization reactor in Section VI. Finally, some conclusions are drawn in Section VII.

Throughout this paper, the following notations are used: $\mathfrak{R}^{n}$ denotes a $n$-dimensional Euclidean space. For any symmetric matrix $P \in \mathfrak{R}^{n \times n}, P>0$ means $P$ is a symmetric positive definite matrix, where the symmetric elements are indicated by "*". The identity vector/matrix and zero vector/matrix with appropriate dimensions are denoted by $\boldsymbol{I}$ and $\mathbf{0}$, respectively.

\section{PRoblem Statement AND PRELIMINARIES}

\section{A. Plant description}

A sampled system of single-input-single-output (SISO) with input delay may be generally described by the following discrete-time controllable canonical state-space model,

$$
\left\{\begin{array}{l}
x(k+1)=\left(A_{\mathrm{m}}+\Delta A\right) x(k)+\left(B_{\mathrm{m}}+\Delta B\right) u(k-d)+\omega(k) \\
y(k)=C_{\mathrm{m}} x(k)
\end{array}\right.
$$

where $x(k), u(k)$ and $y(k)$ denotes the system state, input and output, respectively. The input delay is denoted by $d$, and $\omega(k)$ is external disturbance. Denote by $\left(A_{\mathrm{m}}, B_{\mathrm{m}}, C_{\mathrm{m}}\right)$ the nominal system matrices, and by $\Delta A$ and $\Delta B$ the model uncertainties as described in the norm-bounded form,

$$
\left(\begin{array}{ll}
\Delta A & \Delta B
\end{array}\right)=\sigma E \Delta\left(H_{\mathrm{A}} H_{\mathrm{B}}\right)
$$

where $E, H_{\mathrm{A}}$ and $H_{\mathrm{B}}$ are known matrices of appropriate dimensions, $\Delta$ is an unknown matrix satisfying the norm bounded condition $\Delta^{T} \Delta \leq \boldsymbol{I}$, and $\sigma$ is a positive scalar describing the size of plant uncertainties.

The aim of this paper is to design a PDRC scheme based on only the output measurement for a sampled system described in (1) subject to input delay, while maintaining the closed-loop system robust stability.

\section{B. Discrete-time model-based ESO}

Consider the following $n$-th order system description in discrete-time domain,

$$
\begin{aligned}
y(k+n)+a_{n-1} y(k+n-1)+\cdots+a_{1} y(k+1)+a_{0} y(k) \\
=b_{n-1} u(k+n-1)+\cdots+b_{1} u(k+1)+b_{0} u(k) \\
\quad+f(y(k), u(k), \omega(k))
\end{aligned}
$$

where $f(y, u, \omega)$ denotes a generalized disturbance composed of system uncertainties and external disturbance. The nominal system model may be written into a transfer function form,

$$
P_{\mathrm{n}}(z)=\frac{b_{n-1} z^{n-1}+\cdots+b_{1} z+b_{0}}{z^{n}+a_{n-1} z^{n-1}+\cdots+a_{1} z+a_{0}}
$$

Denote by $x(k)=\left[\begin{array}{lllll}x_{1}^{T}(k) & x_{2}^{T}(k) & \cdots & x_{n}^{T}(k)\end{array}\right]^{T}$ the nominal system state relating to $P_{\mathrm{n}}(z)$. The corresponding state-space realization is expressed by $C_{\mathrm{m}}\left(z \boldsymbol{I}-A_{\mathrm{m}}\right)^{-1} B_{\mathrm{m}}$ with

$$
\begin{gathered}
A_{\mathrm{m}}=\left[\begin{array}{ccccc}
0 & 1 & 0 & \cdots & 0 \\
0 & 0 & 1 & \cdots & 0 \\
\vdots & \vdots & \vdots & \ddots & \vdots \\
0 & 0 & 0 & 0 & 1 \\
-a_{0} & -a_{1} & -a_{2} & \cdots & -a_{n-1}
\end{array}\right], B_{\mathrm{m}}=\left[\begin{array}{c}
0 \\
0 \\
\vdots \\
0 \\
b_{0}
\end{array}\right], \\
C_{\mathrm{m}}=\left[\begin{array}{lllll}
1 & b_{1} / b_{0} & \cdots & b_{n-2} / b_{0} & b_{n-1} / b_{0}
\end{array}\right] .
\end{gathered}
$$

To estimate $f(y, u, \omega)$, an augmented system description is given below by regarding $f$ as an extended state,

$$
\left\{\begin{array}{l}
X(k+1)=\hat{A} X(k)+\hat{B} u(k)+\hat{E} \Delta f(k+1) \\
y(k)=\hat{C} X(k)
\end{array}\right.
$$

where $X(k)=\left[\begin{array}{ll}x^{T}(k) & f^{T}(k)\end{array}\right]^{T}, \Delta f(k+1)=f(k+1)-f(k)$,

$$
\hat{A}=\left[\begin{array}{cc}
A_{\mathrm{m}} & {\left[\begin{array}{c}
\mathbf{0}_{(n-1) \times 1} \\
1 \\
\mathbf{0}_{1 \times n}
\end{array}\right]}
\end{array}\right], \hat{B}=\left[\begin{array}{c}
B_{\mathrm{m}} \\
\mathbf{0}
\end{array}\right], \hat{C}=\left[\begin{array}{c}
C_{\mathrm{m}}^{T} \\
\mathbf{0}
\end{array}\right]^{T}, \hat{E}=\left[\begin{array}{c}
\mathbf{0}_{n \times 1} \\
1
\end{array}\right] .
$$

Based on the augmented system description in (6), a model-based ESO (MESO) is established as

$$
\left\{\begin{array}{l}
\hat{X}(k+1)=\hat{A} \hat{X}(k)+\hat{B} u(k)+L[y(k)-\hat{C} \hat{X}(k)] \\
u(k)=\hat{r}(k)-K \hat{X}(k)
\end{array}\right.
$$

where $L, K$ and $\hat{r}(k)$ denote the observer gain, the controller gain and a modified reference signal, respectively, all of which will be designed in the next section.

Given an input delay denoted by $d$, if the measured output is directly used in the above MESO, it follows that

$$
\left\{\begin{array}{l}
\hat{X}(k+1)=\hat{A} \hat{X}(k)+\hat{B} u(k)+L[y(k-d)-\hat{C} \hat{X}(k)] \\
u(k)=\hat{r}(k)-K \hat{X}(k)
\end{array}\right.
$$

It is obvious that the observation error, $y(k-d)-\hat{C} \hat{X}(k)$, is mismatched in the time step, which may lead to the instability of MESO. To circumvent this issue, an undelayed output should be adopted to replace $y(k-d)$ in (8). This motivates a predictor design for delay-free output prediction to be detailed in the next section.

\section{PROPOSED PDRC}

The proposed PDRC scheme is depicted in Fig. 1, where $P(z)$ denotes the plant that may be stable, integrating or unstable type, $P_{\mathrm{n}}(z)=G_{\mathrm{n}}(z) z^{-d_{\mathrm{n}}}$ the nominal plant model (discretized by a zero-order holder), $G_{\mathrm{n}}(z)$ the delay-free part, $d_{\mathrm{n}}$ the nominal input delay identified by the process modeling, $K$ a feedback controller based on the estimated state given by MESO, $F_{\mathrm{p}}(z)$ a filter in the FSP [4] as outlined by the dash box in Fig. $1, F(z)$ another filter for set-point tracking. 


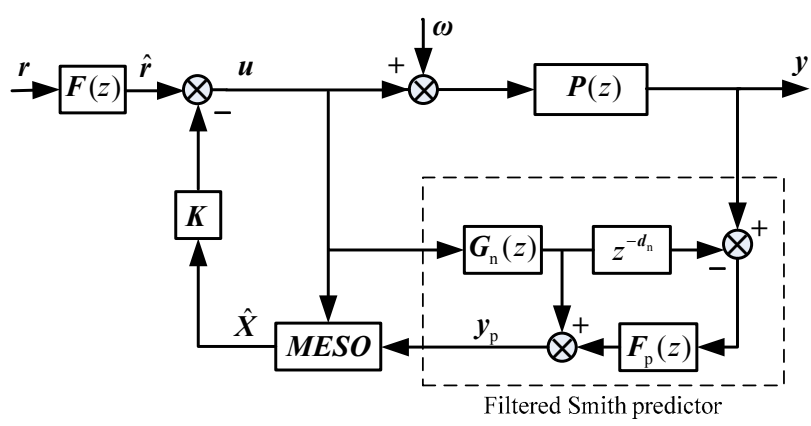

Fig. 1 Block diagram of the proposed PDRC scheme

For clarity, the proposed designs for MESO, $K, F(z)$, and $F_{\mathrm{p}}(z)$ are detailed in the following subsections, respectively.

\section{A. Predictive MESO design}

To estimate the augmented system in (6) without delay, a predictive MESO is proposed as

$$
\left\{\begin{array}{l}
\hat{X}(k+1)=\hat{A} \hat{X}(k)+\hat{B} u(k)+L\left[y_{\mathrm{p}}(k)-\hat{y}(k)\right] \\
\hat{y}(k)=\hat{C} \hat{X}(k)
\end{array}\right.
$$

where $y_{\mathrm{p}}(k)$ denotes the predicted delay-free output, which is exactly the predictor output, as shown in Fig. 1.

For simplicity, the observer gain vector $L$ is determined by deploying the transfer function poles of (9) at a desired location in the z-plane, i.e.,

$$
\Pi_{1}(z)=|z \boldsymbol{I}-(\hat{A}-L \hat{C})|=\left(z-\alpha_{0}\right)^{n+1}
$$

where $\alpha_{0} \in(0,1)$ is a tuning parameter which may be initially taken as 0.5 for practical implementation, corresponding to the observer bandwidth in discrete-time domain.

Accordingly, the observer gain vector is derived by using the Ackerman formula [26] as

$$
L=\Pi_{1}(\hat{A})\left[\begin{array}{llll}
\hat{C}^{T} & (\hat{C} \hat{A})^{T} & \cdots & \left(\hat{C} \hat{A}^{n}\right)^{T}
\end{array}\right]^{-T}\left[\begin{array}{llll}
0 & 0 & \cdots & 1
\end{array}\right]^{T}
$$

\section{B. PDRC controller design}

The control law in the proposed control structure is taken as

$$
u(k)=\hat{r}(k)-K \hat{X}(k)
$$

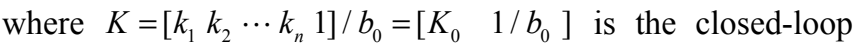
feedback controller to be determined for disturbance rejection, and $\hat{r}(k)$ is a modified reference signal as introduced in (7) for set-point tracking via $F(z)$.

If the generalized disturbance $f(y, u, \omega)$ could be exactly estimated for counteraction, while $\hat{X}(k)$ could also be accurately estimated by $d_{\mathrm{n}}$ step ahead of the current system state, the characteristic equation of the resulting control system by substituting (12) into (6) will become

$$
\Pi_{2}(z)=|z \boldsymbol{I}-(\hat{A}-\hat{B} K)|
$$

which may be reformulated as

$$
\Pi_{2}(z)=(z-1)\left[z^{n}+\left(a_{n-1}+k_{n}\right) z^{n-1}+\cdots+\left(a_{1}+k_{2}\right) z+a_{0}+k_{1}\right] .
$$

To facilitate the controller tuning in practical application, we prescribe the desired closed-loop system poles in the form of

$$
z^{n}+\left(a_{n-1}+k_{n}\right) z^{n-1}+\cdots+\left(a_{1}+k_{2}\right) z+a_{0}+k_{1}=\left(z-\alpha_{\mathrm{c}}\right)^{n}
$$

where $\alpha_{\mathrm{c}} \in(0,1)$ is a tuning parameter which may be initially taken as 0.9 for practical implementation. Therefore, the closed-loop performance for set-point tracking can be conveniently tuned by $\alpha_{\mathrm{c}}$, though the corresponding multiple pole may not be optimal for the closed-loop system.

Accordingly, we have

$$
k_{i}=\left(\begin{array}{c}
n \\
i-1
\end{array}\right)\left(-\alpha_{\mathrm{c}}\right)^{n-i+1}-a_{i-1}, i=1, \ldots, n .
$$

Remark 1. By prescribing the desired multiple pole of MESO and the closed-loop control system for disturbance rejection, respectively, there is only a single tuning parameter in either $L$ of MESO or the PDRC controller $K$. Hence, it is convenient to monotonically tune the single parameter to realize a good trade-off between the state prediction (or control) performance and its robustness.

By taking the z-transform of (9) and (12), we have

$$
\left\{\begin{array}{l}
z \hat{X}(z)=\hat{A} \hat{X}(z)+\hat{B} u(z)+L\left[y_{\mathrm{p}}(z)-\hat{y}(z)\right] \\
u(z)=\hat{r}(z)-K \hat{X}(z)
\end{array}\right.
$$

where $\hat{X}(z), u(z), y_{\mathrm{p}}(z), \hat{y}(z)$, and $\hat{r}(z)$ are the z-transforms of $\hat{X}(k), u(k), y_{\mathrm{p}}(k), \hat{y}(k)$, and $\hat{r}(k)$, respectively.

From (15), we obtain

$$
u(z)=F_{1}(z) \hat{r}(z)-F_{2}(z) y_{\mathrm{p}}(z)
$$

where

$$
\begin{aligned}
F_{1}(z) & =1-K(z \boldsymbol{I}-\hat{A}+\hat{B} K+L \hat{C})^{-1} \hat{B}, \\
F_{2}(z) & =K(z \boldsymbol{I}-\hat{A}+\hat{B} K+L \hat{C})^{-1} L \\
& =\frac{1}{(z-1)} \frac{K \operatorname{adj}\left(z \boldsymbol{I}_{n+1}-\hat{A}+\hat{B} K+L \hat{C}\right) L}{\operatorname{det}\left(z \boldsymbol{I}_{n}-A_{\mathrm{m}}+B_{\mathrm{m}} K_{0} / b_{0}+L_{n} C_{\mathrm{m}}\right)} .
\end{aligned}
$$

where $L_{n}$ denotes the front $n$ rows of $L$. Note that there is a pole at $z=1$, indicating that $F_{2}(z)$ has an integral action independent of the MESO design.

Therefore, the above MESO and $K$ can be implemented by $F_{1}(z)$ and $F_{2}(z)$ in practice. That is to say, the proposed PDRC scheme is equivalent to a predictor-based two-degree-of-freedom (2DOF) control structure depicted in Fig. 2.

For set-point tracking, to ensure no steady-state output error, a modified reference signal is introduced as shown in Fig. 2 as

$$
\hat{r}(z)=F(z) r(z)
$$

where $F(z)$ is a pre-filter of the set-point.

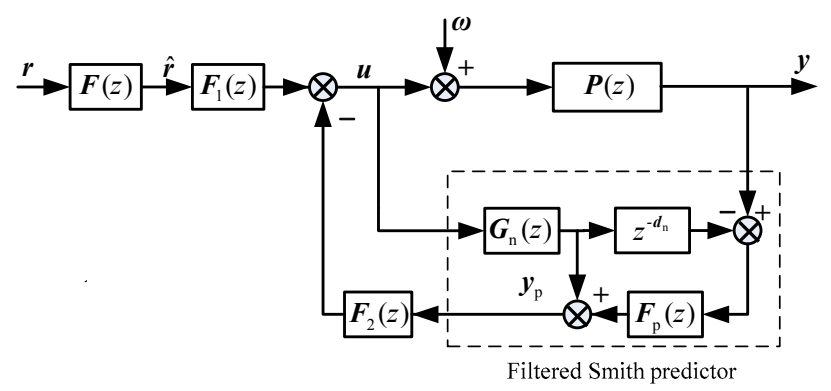

Fig. 2 Equivalent representation of the proposed PDRC scheme

If $\hat{X}(k)$ could be accurately estimated by $d_{\mathrm{n}}$ step ahead of the current system state, the set-point response transfer function will become 


$$
\begin{aligned}
y(z) & =C_{\mathrm{m}}\left(z \boldsymbol{I}-A_{\mathrm{m}}+B_{\mathrm{m}} K_{0}\right)^{-1} B_{\mathrm{m}} z^{-d_{\mathrm{n}}} F(z) r(z) \\
& =T_{\mathrm{d}}(z) F(z) z^{-d_{\mathrm{n}}} r(z)
\end{aligned}
$$

Denote $T_{\mathrm{d}}(z)=C_{\mathrm{m}}\left(z \boldsymbol{I}-A_{\mathrm{m}}+B_{\mathrm{m}} K_{0}\right)^{-1} B_{\mathrm{m}}$. Ideally, it is desired to let $T_{\mathrm{d}}(z) F(z)=1$ for set-point tracking. However, it is impractical to take $F(z)=1 / T_{\mathrm{d}}(z)$ due to physical improperness.

Note that to realize no steady-state tracking error, the following steady-state constraint must be satisfied,

$$
\lim _{z \rightarrow 1} F(z)=\lim _{z \rightarrow 1} \frac{1}{T_{\mathrm{d}}(z)}=\frac{1}{C_{\mathrm{m}}\left(\boldsymbol{I}-A_{\mathrm{m}}+B_{\mathrm{m}} K_{0}\right)^{-1} B_{\mathrm{m}}}
$$

It is therefore proposed to design the pre-filter in the form of

$$
F(z)=\frac{K_{\mathrm{f}}(1-\lambda)^{h} z^{h}}{(z-\lambda)^{h}}
$$

where $\lambda$ is a tuning parameter, $h$ is the filter order that may be practically specified to comply with the control limit for implementation, and

$$
K_{\mathrm{f}}=\frac{1}{C_{\mathrm{m}}\left(\boldsymbol{I}-A_{\mathrm{m}}+B_{\mathrm{m}} K_{0}\right)^{-1} B_{\mathrm{m}}}
$$

Hence, both the set-point tracking and disturbance rejection can be separately optimized in the proposed control scheme, thus facilitating practical applications for system performance optimization.

\section{Design of the FSP filter}

For the convenience of sampling implementation, it is proposed to take the FSP filter in the following form,

$$
F_{\mathrm{p}}(z)=\frac{\left(1-\lambda_{\mathrm{f}}\right)^{l+1}}{\left(z-\lambda_{\mathrm{f}}\right)^{l+1}} \sum_{i=1}^{l+1} \beta_{i} z^{i}, \sum_{i=1}^{l+1} \beta_{i}=1
$$

where $\lambda_{\mathrm{f}} \in(0,1)$ is a tuning parameter, $\beta_{i}(i=0,1, \ldots, l$. $)$ are the filter parameters to be determined, $l$ is the number of the plant model poles located close to, on, or outside the unit circle in the z-plane. Note that a unified design of the filter is given in (24) is for application to stable, integral and unstable processes, compared to different choices of the filter in [4] with respect to different process models in frequency domain.

It can be easily seen from (24) that

$$
\lim _{z \rightarrow 1} F_{\mathrm{p}}(z)=1
$$

which ensures no steady-state prediction error.

To guarantee internal stability of the closed-loop system for any plant of stable, integrating, or unstable type, an equivalent control structure is preferred for practical implementation, which is shown in Fig. 3.

It follows that

$$
F_{\mathrm{d}}(z)=G_{\mathrm{n}}(z)\left[1-z^{-d_{\mathrm{n}}} F_{\mathrm{p}}(z)\right]
$$

which should be designed stable for implementation in practice.

Denote by $\bar{y}$ the real undelayed output. It can be derived from Fig. 3 that the undelayed output prediction error under perfect model match is in the form of

$$
\bar{e}(z)=\bar{y}-y_{\mathrm{p}}=F_{\mathrm{d}}(z) \omega(z)
$$

Owing to $\lim _{z \rightarrow 1} F_{\mathrm{d}}(z)=0$ as can be verified from (26), the output prediction error in (27) vanishes in the steady state, which means that static or asymptotically stable load disturbance would not result in steady-state prediction error. Furthermore, the form

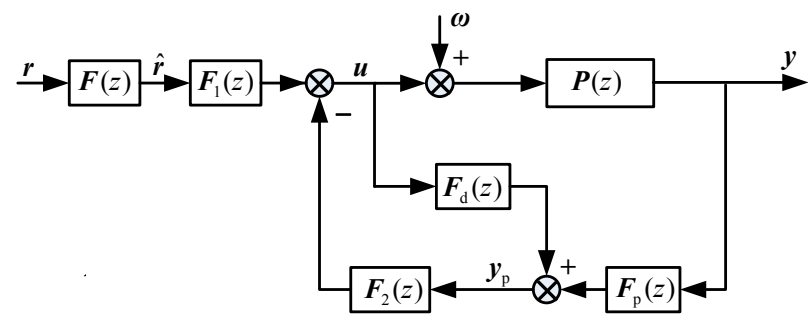

Fig. 3 An equivalent control structure for implementation

of $F_{\mathrm{p}}(z)$ implies that a smaller value of $\lambda_{\mathrm{f}}$ will lead to faster prediction of output error arising from load disturbance.

Note that the delay-free part of the plant model, $G_{\mathrm{n}}(z)$, is included in $F_{\mathrm{d}}(z)$. If there is any integrating $(z=1)$ or unstable pole $(|z|>1)$ in $G_{\mathrm{n}}(z)$, the corresponding $F_{\mathrm{d}}(z)$ will become unstable. Besides, if there exists any slow pole $(|z| \rightarrow 1)$ in $G_{\mathrm{n}}(z)$, the prediction performance will be slowed down. To circumvent the above problem, the following constraints are imposed to design $F_{\mathrm{p}}(z)$,

$$
\begin{aligned}
& 1-\left.z^{-d_{\mathrm{n}}} F_{\mathrm{p}}(z)\right|_{z=z_{i}, i=1, \ldots, s_{1}}=0 \\
& \left.\frac{d^{s_{2}}}{d z^{s_{2}}}\left[1-z^{-d_{\mathrm{n}}} F_{\mathrm{p}}(z)\right]\right|_{z=1}=0
\end{aligned}
$$

where $z_{i}, i=1, \cdots s_{1}$ are slow poles (close to the unit circle in the z-plane) or unstable poles (outside the unit circle) in $G_{\mathrm{n}}(z)$ for a stable or unstable process, $s_{2}$ is the number of integrating poles $(z=1)$ for an integrating process.

If there is no slow pole in $G_{\mathrm{n}}(z)$, the prediction filter is designed in terms of (24) as

$$
F_{\mathrm{p}}(z)=\frac{\left(1-\lambda_{\mathrm{f}}\right)^{q}}{\left(z-\lambda_{\mathrm{f}}\right)^{q}}
$$

where $\lambda_{\mathrm{f}} \in(0,1)$ is a user-specified tuning parameter to obtain a good trade-off between the prediction performance and its robustness against process uncertainties, which may be initially taken as 0.9 for practical implementation.

When there exist slow poles, by substituting (24) into (28), the filter parameters, $\beta_{i}\left(i=0,1, \ldots, s_{1}.\right)$ can be determined.

\section{ROBUST STABILITY ANALYSIS}

Considering the equivalent structure of the proposed control scheme as shown in Fig. 3, the state-space representations of the main components are written, respectively, as

$$
\begin{aligned}
& P(z):\left\{\begin{array}{l}
x(k+1)=\tilde{A} x(k)+\tilde{B} u(k-d) \\
y(k)=C_{\mathrm{m}} x(k)
\end{array}\right. \\
& F_{\mathrm{d}}(z):\left\{\begin{array}{l}
x_{\mathrm{s}}(k+1)=A_{\mathrm{s}} x_{\mathrm{s}}(k)+B_{\mathrm{s}} u(k) \\
y_{\mathrm{s}}(k)=C_{\mathrm{s}} x_{\mathrm{s}}(k)
\end{array}\right. \\
& F_{\mathrm{p}}(z):\left\{\begin{array}{l}
x_{\mathrm{f}}(k+1)=A_{\mathrm{f}} x_{\mathrm{f}}(k)+B_{\mathrm{f}} y(k) \\
y_{\mathrm{f}}(k)=C_{\mathrm{f}} x_{\mathrm{f}}(k)+D_{\mathrm{f}} y(k)
\end{array}\right. \\
& F_{2}(z):\left\{\begin{array}{l}
x_{\mathrm{c}}(k+1)=A_{\mathrm{c}} x_{\mathrm{c}}(k)+B_{\mathrm{c}} y_{\mathrm{p}}(k) \\
u(k)=-C_{\mathrm{c}} x_{\mathrm{c}}(k)
\end{array}\right.
\end{aligned}
$$

where $\tilde{A}=A_{\mathrm{m}}+\Delta A, \tilde{B}=B_{\mathrm{m}}+\Delta B, y_{\mathrm{p}}(k)=y_{\mathrm{s}}(k)+y_{\mathrm{f}}(k)$.

By using algebraic manipulations, the closed-loop system description is obtained as

$$
\bar{x}(k+1)=\left(\bar{A}+\sigma \bar{E} \Delta \bar{H}_{\mathrm{A}}\right) \bar{x}(k)+\left(\bar{A}_{\mathrm{d}}+\sigma \bar{E} \Delta \bar{H}_{\mathrm{B}}\right) \bar{x}(k-d)
$$


where $\bar{x}(k) \triangleq\left[\begin{array}{llll}x^{T}(k) & x_{\mathrm{s}}^{T}(k) & x_{\mathrm{f}}^{T}(k) & x_{\mathrm{c}}^{T}(k)\end{array}\right]^{T}$,

$\bar{A}=\left[\begin{array}{cccc}A_{\mathrm{m}} & \mathbf{0} & \mathbf{0} & \mathbf{0} \\ \mathbf{0} & A_{\mathrm{s}} & \mathbf{0} & -B_{\mathrm{s}} C_{\mathrm{c}} \\ B_{\mathrm{f}} C_{\mathrm{m}} & \mathbf{0} & A_{\mathrm{f}} & \mathbf{0} \\ B_{\mathrm{c}} D_{\mathrm{f}} C_{\mathrm{m}} & B_{\mathrm{c}} C_{\mathrm{s}} & B_{\mathrm{c}} C_{\mathrm{f}} & A_{\mathrm{c}}\end{array}\right], \bar{A}_{\mathrm{d}}=\left[\begin{array}{cccc}\mathbf{0} & \mathbf{0} & \mathbf{0} & -B_{\mathrm{m}} C_{\mathrm{c}} \\ \mathbf{0} & \mathbf{0} & \mathbf{0} & \mathbf{0} \\ \mathbf{0} & \mathbf{0} & \mathbf{0} & \mathbf{0} \\ \mathbf{0} & \mathbf{0} & \mathbf{0} & \mathbf{0}\end{array}\right]$,

$\bar{E}=\left[\begin{array}{llll}E^{T} & \mathbf{0} & \mathbf{0} & \mathbf{0}\end{array}\right]^{T}, \bar{H}_{\mathrm{A}}=\left[\begin{array}{llll}H_{\mathrm{A}} & \mathbf{0} & \mathbf{0} & \mathbf{0}\end{array}\right], \bar{H}_{\mathrm{B}}=\left[\begin{array}{llll}\mathbf{0} & \mathbf{0} & \mathbf{0} & -H_{\mathrm{B}} C_{\mathrm{c}}\end{array}\right]$.

Before analyzing the robust stability condition, the following two lemmas are briefly presented.

Lemma 1 [27]. Given a symmetric positive definite matrix $\Lambda$, any sequence of discrete-time variable $x$ in $[-d, 0] \cap \mathrm{Z} \rightarrow \Re^{n}$ with $d>1$ satisfies the following inequality,

$$
-\sum_{i=k-d}^{k-1} y^{T}(i) \Lambda y(i) \leq-\frac{1}{d}\left[\begin{array}{l}
\vartheta_{1} \\
\vartheta_{2}
\end{array}\right]^{T}\left[\begin{array}{cc}
\Lambda & \mathbf{0} \\
\mathbf{0} & 3 \Lambda
\end{array}\right]\left[\begin{array}{l}
\vartheta_{1} \\
\vartheta_{2}
\end{array}\right]
$$

where $y(i)=x(i+1)-x(i), \vartheta_{1}=x(k)-x(k-d)$, and

$\vartheta_{2}=x(k)+x(k-d)-\frac{2}{d+1} \sum_{i=k-d}^{k} x(i)$

Lemma 2 [28]. Given matrices $X$ and $Y$, with appropriate dimensions, the following inequality holds for any scalar $\varepsilon>0$ and matrix $\Delta$ that satisfies $\Delta^{T} \Delta \leq \boldsymbol{I}$,

$$
X \Delta Y+Y^{T} \Delta^{T} X^{T} \leq \varepsilon X X^{T}+\varepsilon^{-1} Y^{T} Y
$$

Now we are in the position to give a sufficient condition which guarantees internal stability of the closed-loop system described in (35).

Theorem 1 . The control system in (35) is asymptotically stable if there exist symmetric matrices $P, Q, R>0$ and scalar $\mu>0$ such that the following LMI holds,

$$
\left[\begin{array}{cccc}
-\Lambda_{1}^{T} P \Lambda_{1}+\bar{Q}-\Lambda_{4}^{T} \bar{R} \Lambda_{4}+\hat{H}^{T} \hat{H} & \Lambda_{2}^{T} P & d \Lambda_{3}^{T} R & \mathbf{0} \\
* & -P & \mathbf{0} & P \hat{E} \\
* & * & -R & d R \bar{E} \\
* & * & * & -\mu \boldsymbol{I}
\end{array}\right]<0
$$

where $\bar{Q} \triangleq \operatorname{diag}\{Q, \quad-Q, \quad \mathbf{0}\}, \bar{R} \triangleq \operatorname{diag}\{R, \quad 3 R\}, \hat{E} \triangleq\left[\begin{array}{ll}\bar{E}^{T} & \mathbf{0}\end{array}\right]^{T}$, $\hat{H} \triangleq\left[\begin{array}{lll}\bar{H}_{\mathrm{A}} & \bar{H}_{\mathrm{B}} & \mathbf{0}\end{array}\right]$,

$$
\begin{aligned}
& \Lambda_{1} \triangleq\left[\begin{array}{ccc}
\boldsymbol{I} & \mathbf{0} & \mathbf{0} \\
-\boldsymbol{I} & \mathbf{0} & (d+1) \boldsymbol{I}
\end{array}\right], \Lambda_{2} \triangleq\left[\begin{array}{ccc}
\bar{A} & \bar{A}_{\mathrm{d}} & \mathbf{0} \\
\mathbf{0} & -\boldsymbol{I} & (d+1) \boldsymbol{I}
\end{array}\right], \\
& \Lambda_{3} \triangleq\left[\begin{array}{lll}
\bar{A}-I & \bar{A}_{\mathrm{d}} & \mathbf{0}
\end{array}\right], \Lambda_{4} \triangleq\left[\begin{array}{ccc}
\boldsymbol{I} & -\boldsymbol{I} & \mathbf{0} \\
\boldsymbol{I} & \boldsymbol{I} & -2 \boldsymbol{I}
\end{array}\right] .
\end{aligned}
$$

with the process uncertainty bound indicated by $\sigma=\sqrt{1 / \mu}$.

Proof: The following Lyapunov-Krasovskii functional candidate is adopted,

$$
V(t)=V_{1}(k)+V_{2}(k)+V_{3}(k)
$$

where

$$
\begin{aligned}
& V_{1}(k)=\xi^{T}(k) \hat{P} \xi(k), \xi(k)=\left[\begin{array}{ll}
\bar{x}^{T}(k) & \sum_{i=k-d}^{k-1} \bar{x}^{T}(i)
\end{array}\right]^{T}, \\
& V_{2}(k)=\sum_{i=k-d}^{k-1} \bar{x}^{T}(i) \hat{Q} \bar{x}(i), V_{3}(k)=d \sum_{i=-d}^{-1} \sum_{j=k+i}^{k-1} \eta^{T}(j) \hat{R} \eta(j), \\
& \eta(j) \triangleq \bar{x}(j+1)-\bar{x}(j) .
\end{aligned}
$$

We define

$$
v(k) \triangleq \frac{1}{d+1} \sum_{i=k-d}^{k} \bar{x}(i), \varphi(k) \triangleq\left[\begin{array}{ccc}
\bar{x}^{T}(k) & \bar{x}^{T}(k-d) & v^{T}(k)
\end{array}\right]^{T}
$$

It follows that

$$
\xi(k)=\Lambda_{1} \varphi(k), \xi(k+1)=\left(\Lambda_{2}+\sigma \hat{E} \Delta \hat{H}\right) \varphi(k) \triangleq \tilde{\Lambda}_{2} \varphi(k) .
$$

Taking the forward difference of $V(t)$ yields

$$
\begin{gathered}
\Delta V_{1}(k)=\varphi^{T}(k)\left(\tilde{\Lambda}_{2}^{T} \hat{P} \tilde{\Lambda}_{2}-\Lambda_{1}^{T} \hat{P} \Lambda_{1}\right) \varphi(k), \\
\Delta V_{2}(k)=\varphi^{T}(k) \tilde{Q} \varphi(k), \tilde{Q}=\operatorname{diag}\{\hat{Q}, \quad-\hat{Q}, \quad \mathbf{0}\}, \\
\Delta V_{3}(k)=d^{2} \varphi^{T}(k) \tilde{\Lambda}_{3}^{T} \hat{R} \tilde{\Lambda}_{3} \varphi(k)-d \sum_{i=k-d}^{k-1} \eta^{T}(i) \hat{R} \eta(i) .
\end{gathered}
$$

where $\tilde{\Lambda}_{3} \triangleq \Lambda_{3}+\sigma \bar{E} \Delta \hat{H}$.

Using the Wirtinger inequality in Lemma 1 , we have

$$
-d \sum_{i=k-d}^{k-1} \eta^{T}(i) \hat{R} \eta(i) \leq-\left[\begin{array}{l}
\theta_{1} \\
\theta_{2}
\end{array}\right]^{T} \tilde{R}\left[\begin{array}{l}
\theta_{1} \\
\theta_{2}
\end{array}\right], \tilde{R}=\operatorname{diag}\{\hat{R}, \quad 3 \hat{R}\} .
$$

where

$$
\left[\begin{array}{l}
\theta_{1} \\
\theta_{2}
\end{array}\right]=\left[\begin{array}{c}
\bar{x}(k)-\bar{x}(k-d) \\
\bar{x}(k)+\bar{x}(k-d)-\frac{2}{d+1} \sum_{i=k-d}^{k} \bar{x}(i)
\end{array}\right] \triangleq \Lambda_{4} \varphi(k)
$$

Therefore, it can be derived that the forward difference of $V(k)$ is bounded by

$$
\Delta V(k) \leq \varphi^{T}(k)\left(\tilde{\Lambda}_{2}^{T} \hat{P} \tilde{\Lambda}_{2}-\Lambda_{1}^{T} \hat{P} \Lambda_{1}+\tilde{Q}+d^{2} \tilde{\Lambda}_{3}^{T} \hat{R} \tilde{\Lambda}_{3}-\Lambda_{4}^{T} \tilde{R} \Lambda_{4}\right) \varphi(k)
$$

Then by Schur complement and Lemma 2, we obtain $\Delta V(t)<0$ if the following matrix inequality condition holds,

$$
\left[\begin{array}{cccc}
-\Lambda_{1}^{T} \hat{P} \Lambda_{1}+\tilde{Q}-\Lambda_{4}^{T} \tilde{R} \Lambda_{4}+\varepsilon \hat{H}^{T} \hat{H} & \Lambda_{2}^{T} \hat{P} & d \Lambda_{3}^{T} \hat{R} & \mathbf{0} \\
* & -\hat{P} & \mathbf{0} & \sigma \hat{P} \hat{E} \\
* & * & -\hat{R} & \sigma d \hat{R} \bar{E} \\
* & * & * & -\varepsilon \boldsymbol{I}
\end{array}\right]<0
$$

Redefining the variables, $P=\hat{P} / \varepsilon, Q=\hat{Q} / \varepsilon, R=\hat{R} / \varepsilon$

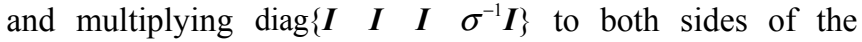
matrix inequality in (40), the LMI condition in (38) follows with $\mu=\sigma^{-2}$. The proof is completed.

To assess the permitted upper bound of process uncertainties, the following optimization procedure can be performed,

$$
\text { Minimize } \mu
$$

$$
\text { s.t. (38) }
$$

Remark 2. The LMI stability condition in (38) can be numerically solved by using any mathematical software package such as the MATLAB Toolbox. The number of variables in (38) is $3 n_{a}^{2}+2 n_{a}+1$, where $n_{a}$ is the dimension of the augmented state defined in (35). Although the computation complexity of (38) is associated with the delay length, it can be solved off-line for the purpose of evaluation.

\section{ILLUSTRATION}

Consider a benchmark example studied in the recent reference [24],

$$
P(s)=G(s) e^{-\tau s}=\frac{b}{s+a} e^{-s s}
$$

In case $a>0$, the example is a stable process. When $a=0$, the example becomes an integrating process. If $a<0$, the example is an unstable process. With a sampling period, $T_{\mathrm{s}}=0.5(\mathrm{~s})$, the correspondingly discretized models are 
obtained in terms of $b=1$ as listed in Table I for different cases of $a=0.05,0$ and -0.05 , respectively.

To perform the control test as done in [24], a unity step change is added to the set-point at $t=0(\mathrm{~s})$, and then a load disturbance with a magnitude of 0.1 is added to the plant input at $t=80(\mathrm{~s})$. For fair comparison in terms of the similar rising speed of the set-point response and the similar disturbance response peak, the observer and controller settings in the proposed method are listed in Table II for different cases, where the pre-filter $F(z)$ is designed by using the formula in (22) with $h=0$, and the FSP filter is designed by using the formula $=$ in (24) with $l=1$. The predictive ADRC in [25] is also performed by taking the bandwidths of the observer and controller as $\omega_{0}=0.77$ and $\omega_{\mathrm{c}}=0.91$ for the stable case, $\omega_{0}=0.76$ and $\omega_{\mathrm{c}}=0.95$ for the integrating case, $\omega_{0}=0.76$ and $\omega_{\mathrm{c}}=0.96$ for the unstable case for comparison. Besides, the FSP control method in [4] is performed, where the corresponding discrete-time controllers for implementation with the same sampling period are listed in Table III according to the control design formulae given therein.

TABLE I

DISCRETE-TIME MODELS FOR DIFFERENT CASES

\begin{tabular}{c|c|c|c}
\hline \hline & \multicolumn{3}{|c}{$b=1$} \\
\cline { 2 - 4 } & $a=0.05$ & $a=0$ & $a=-0.05$ \\
\hline $\begin{array}{c}\text { Discrete-time model } \\
G(z)\end{array}$ & $\frac{0.4938}{z-0.9753}$ & $\frac{0.5}{z-1}$ & $\frac{0.5063}{z-1.025}$ \\
\hline \hline
\end{tabular}

TABLE ॥

PROPOSED OBSERVER AND CONTROLLER SETTINGS

\begin{tabular}{c|c|c|c}
\hline \hline & $a=0.05$ & $a=0$ & $a=-0.05$ \\
\hline$\alpha_{0}$ & 0.25 & 0.48 & 0.45 \\
\hline$\alpha_{c}$ & 0.93 & 0.95 & 0.95 \\
\hline$F$ & 0.1418 & 0.1 & 0.0988 \\
\hline$F_{1}$ & $\frac{(z-0.25)^{2}}{(z-1)(z+0.5453)}$ & $\frac{(z-0.48)^{2}}{(z-1)(z+0.09)}$ & $\frac{(z-0.45)^{2}}{(z-1)(z+0.175)}$ \\
\hline$F_{2}$ & $\frac{1.2745(z-0.9374)}{(z-1)(z+0.5453)}$ & $\frac{0.6448(z-0.9581)}{(z-1)(z+0.09)}$ & $\frac{0.76412(z-0.9609)}{(z-1)(z+0.175)}$ \\
\hline$F_{\mathrm{p}}$ & $\frac{0.16027 z(z-0.9601)}{(z-0.92)^{2}}$ & $\frac{0.1841 z(z-0.9734)}{(z-0.93)^{2}}$ & $\frac{0.14784 z(z-0.9892)}{(z-0.96)^{2}}$ \\
\hline \hline
\end{tabular}

TABLE III

DISCRETIZED CONTROLLER SETTINGS IN THE FSP METHOD [4]

\begin{tabular}{c|c|c|c}
\hline \hline & $F_{\mathrm{FSP}}(\mathrm{z})$ & $C_{\mathrm{FSP}}(z)$ & $F_{\mathrm{r}}$ \\
\hline$a=0.05$ & 1 & $\frac{0.1333 z-0.13}{z-1}$ & $\frac{1.6866(z-0.9663)(z-0.9355)}{(z-0.9394)^{2}}$ \\
\hline$a=0$ & 1 & 0.1 & $\frac{0.9525 z(\mathrm{z}-0.9764)}{(\mathrm{z}-0.85)^{2}}$ \\
\hline$a=-0.05$ & $\frac{0.25 z-0.2253}{z-0.9753}$ & $\frac{0.2 z-0.195}{z-1}$ & $\frac{0.96142(z-0.9882)(\mathrm{z}-0.9048)^{2}}{(\mathrm{z}-0.9753)(\mathrm{z}-0.9355)^{2}}$ \\
\hline \hline
\end{tabular}

The control results are shown in Figs. 4-6 for the input delay $\tau=5$, i.e., $d=10$ in discrete-time domain. It is seen that the disturbance rejection performance is evidently improved by the proposed method. Note that a faster set-point tracking speed and smaller disturbance response peak can be conveniently obtained by monotonically decreasing the tuning parameters in the proposed controllers, which is omitted for brevity.

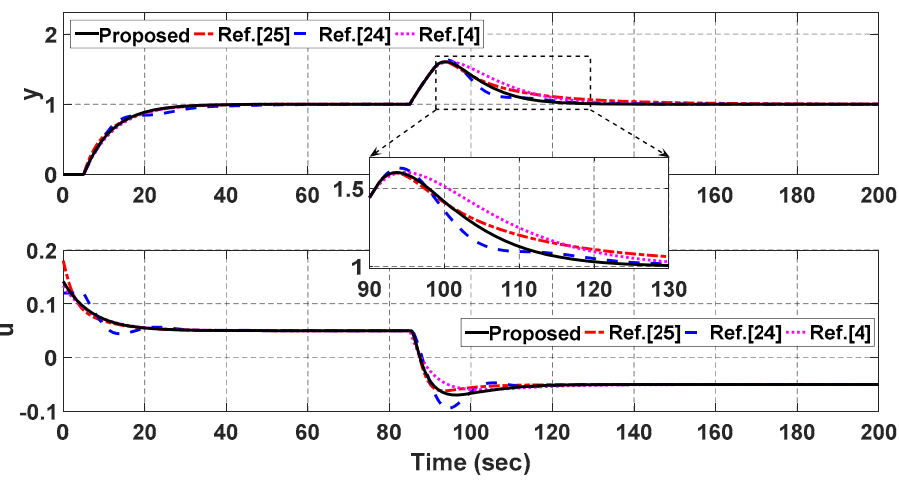

Fig. 4 Control results for the stable process

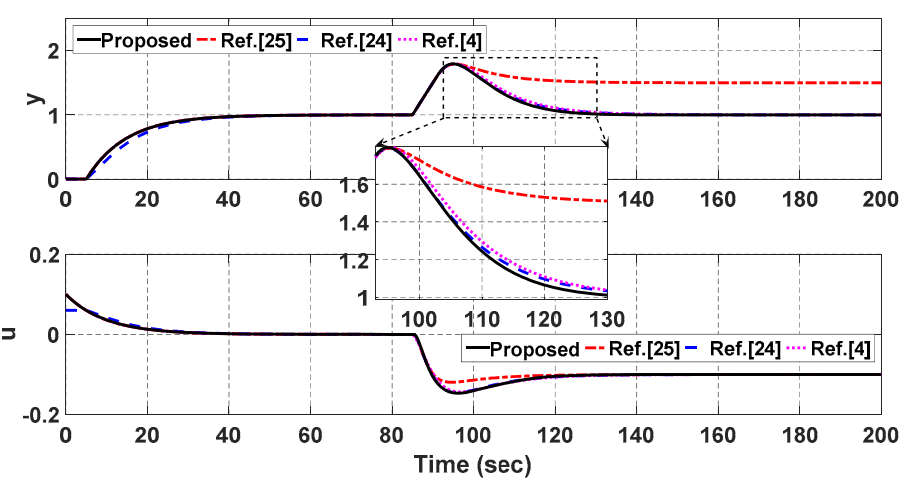

Fig. 5 Control results for the integrating process
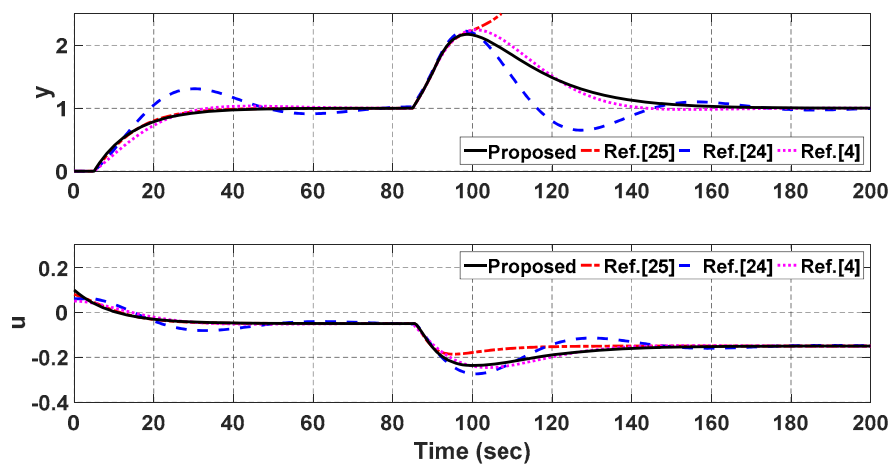

Fig. 6 Control results for the unstable process
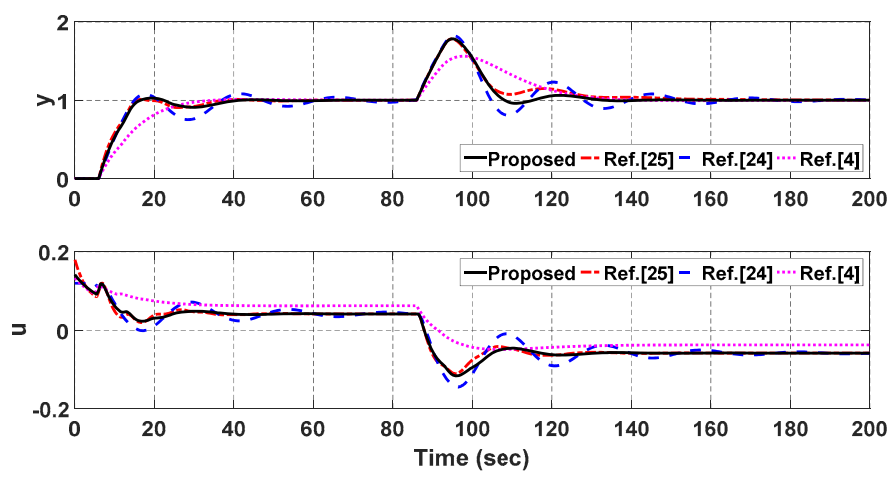

Fig. 7 Control results for the perturbed stable process 
Then assume that in the stable case the process gain and time delay are actually $20 \%$ larger than the model, and in the unstable case, the time delay is actually 50\% larger. Figs. 7 and 8 show the perturbed output responses, respectively. It is seen that the proposed method maintains good robustness compared with the cited methods. Note that by solving the LMI stability condition in (38), the maximum delay uncertainty for the unstable case is $50 \%$ larger than the nominal delay, well verified by the simulation result in Fig. 8 .
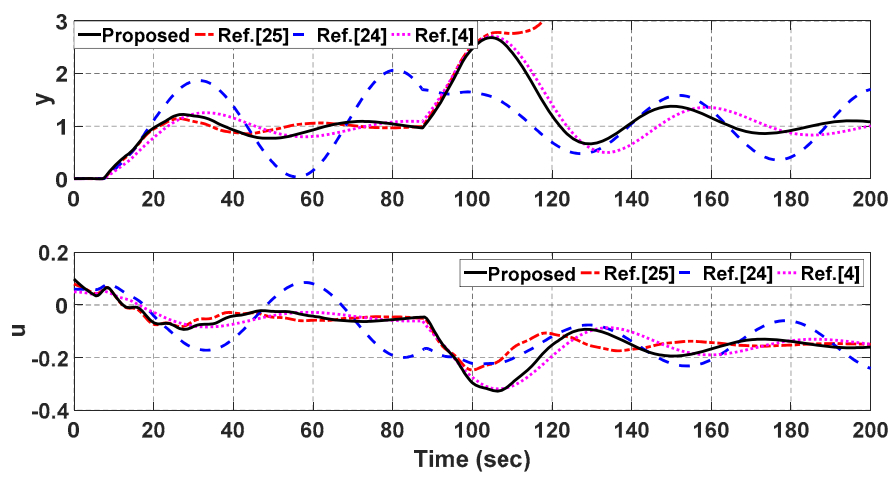

Fig. 8 Control results for the perturbed unstable process

\section{EXPERIMENTAL RESULT}

A temperature control system for a 4-litre crystallization reactor containing 2-litre aqueous solution is used for experiment, which is shown in Fig. 9.

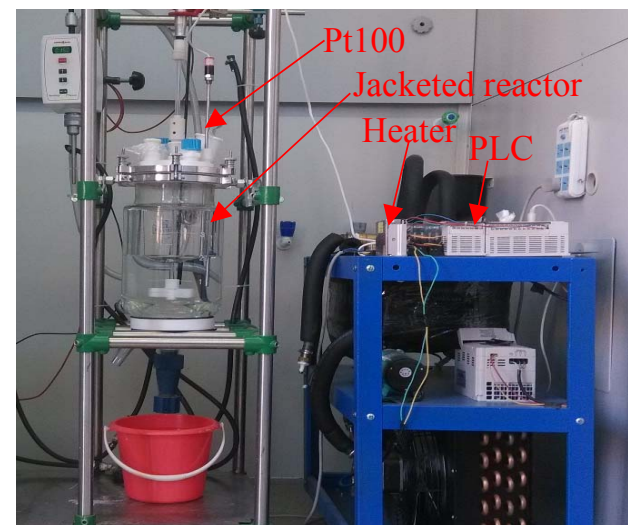

Fig. 9 Temperature control system for a 4-litre jacketed reactor

The temperature control system consists of a 7-litre thermostatic circulator filled with ethylene glycol and distilled water in proportion of $2: 3$, an electric heater with a capacity of 2000 (W) regulated via a zero-crossing solid state relay with pulse-width modulation, a PT100 thermocouple, a programmable-logic-controller (PLC) made by Siemens company, and a 64-bit data acquisition card (AT-MIO-64X) of National Instruments used for analog-to-digital and digital-to-analog conversions. The control task is specified as quickly heating up the reactor solution from the room temperature $\left(25^{\circ} \mathrm{C}\right)$ to $55^{\circ} \mathrm{C}$ for preparing the L-glutamic acid crystallization, and then maintaining the operation temperature of $55^{\circ} \mathrm{C}$ against load disturbance (e.g., feeding the raw solute and solution for operating the crystallization process).
By performing an open-loop step response test, that is, heating up the reactor solution by fully turning on the heater from the room temperature $\left(25^{\circ} \mathrm{C}\right)$ to $55^{\circ} \mathrm{C}$, the following transfer function model for the temperature response is obtained by using the step response identification method [2],

$$
P(s)=\frac{0.0004325}{s(663.4 s+1)} e^{-99 s}
$$

Owing to the slow dynamics of the temperature response, the sampling period is taken as $T_{\mathrm{s}}=3(\mathrm{~s})$ for control implementation. Correspondingly, a discrete-time model is obtained as

$$
P(z)=\frac{2.9291 \times 10^{-6}(z+0.9985)}{(z-1)(z-0.9955)} z^{-33}
$$

A control test is performed to heat up the reactor solution from $25^{\circ} \mathrm{C}$ to $55^{\circ} \mathrm{C}$. A load disturbance is imposed by adding $200(\mathrm{ml})$ solution of distilled water with the room temperature into the reactor after the solution temperature has reached $55^{\circ} \mathrm{C}$.

In the proposed method, the tuning parameters are taken as $\alpha_{0}=0.9952$ and $\alpha_{\mathrm{c}}=0.9923$ with $b_{0}=2.9247 \times 10^{-6}$. The set-point pre-filter is designed by using the formula in (22) as $F(z)=0.1036 z /(z-0.989)$ with $h=1$ to comply with the heating power for control implementation, and the FSP filter is designed by using the formula in (24) with $l=1$ as

$$
F_{\mathrm{p}}(z)=\frac{0.3648 z(z-0.9825)}{(z-0.92)^{2}}
$$

For comparison, the recently developed IMC-based ADRC method [23] is also performed by specifying the observer bandwidth $\omega_{0}=0.0018$ and the controller bandwidth $\omega_{\mathrm{c}}=0.0085$ according to the tuning guideline given therein. In addition, the filtered SP control method given in [4] is performed, which had demonstrated superiority over previous SP or DTC methods for control of integrating processes with input delay. The controllers are taken in terms of the design formulae given therein as

$$
F(s)=\frac{1}{(335 s+1)^{2}}, C(s)=\frac{3567 s+5.377}{0.6634 s+1}, F_{\mathrm{r}}(s)=\frac{1.8188 \times 10^{3} s+1}{(430 s+1)^{3}} .
$$

The experimental results along with the control signals of heating power are shown in Figs. 10 and 11, respectively.

It is seen that fast heating-up response without overshoot is obtained by the proposed method, together with better disturbance rejection performance, compared to the IMC-based ADRC method [23] and the filtered SP control method [4]. In particular, more than 30 minutes are saved for recovering the solution temperature to the operating temperature zone of $(55.0 \pm 0.2)^{\circ} \mathrm{C}$ against the load disturbance of feeding raw materials, compared to the ADRC method [23]. Note that any negative control signal (i.e., $u<0$ ) could not be implemented due to no cooling function of the thermostatic circulator. If the ADRC method [23] or the filtered SP control method [4] is tuned to yield further aggressive control action to expedite the set-point tracking or load disturbance rejection, the solution temperature will not recover to the operating temperature of $55^{\circ} \mathrm{C}$, i.e., a steady-state temperature deviation will be turned out, because negative control signal (corresponding to the cooling action) will be required, which in fact cannot be implemented due to the control limit of $0 \leq u \leq 100$. Such 
control invalidity indicated by $u=0$ at certain moments is shown in Fig. 11.

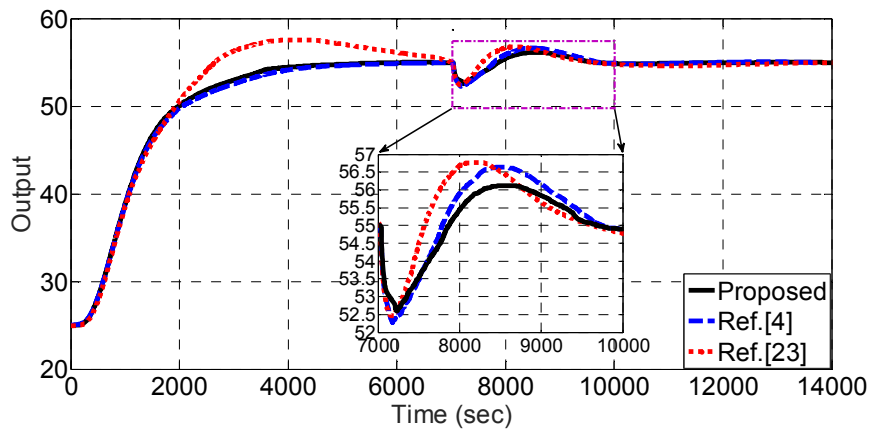

Fig. 10 Temperature response by using three control methods

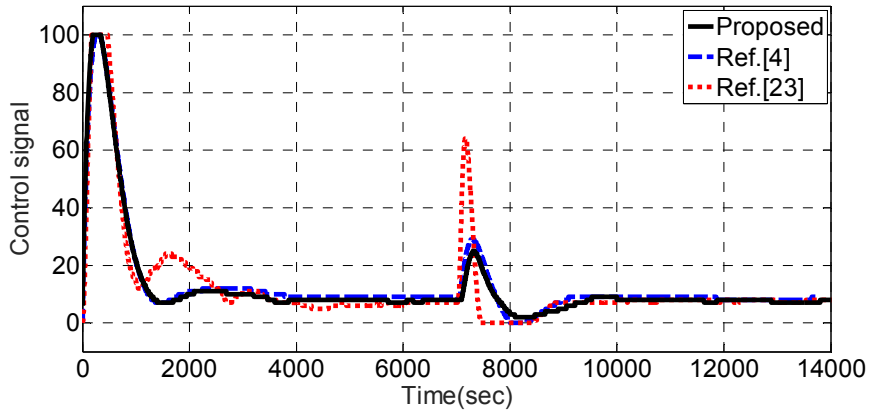

Fig. 11 Control signals for implementation

\section{CONCLUSIONS}

A PDRC scheme has been proposed for sampled systems with input delay, which can be used for open-loop stable, integrating, and unstable processes. A predictive MESO is introduced based on using the FSP for delay-free output prediction, which facilitates the control design using only output measurement. By specifying the desired transfer function poles of the predictive MESO and the closed-loop control system, the MESO gain vector and the PDRC controller are analytically derived. In fact, the proposed PRDC scheme can be equivalently transformed into a predictor-based $2 \mathrm{DOF}$ control scheme for implementation. A noteworthy merit is that there is a single tuning parameter in the proposed MESO, FSP, and PRDC controller, respectively, which can be monotonically tuned to meet a good trade-off between the prediction (or control) performance and its robustness. A sufficient condition for holding robust stability of the closed-loop system is established in terms of LMI, by using the discrete-time Wirtinger inequality. The stability condition may be used to assess the upper bound of process uncertainties allowed for control implementation. A benchmark example has well demonstrated the advantage of the proposed control method in comparison with the recently developed ADRC and FSP based control methods. The application to a temperature control system of a 4-litre crystallization reactor has verified the effectiveness and merit of the proposed control scheme.

\section{REFERENCES}

[1] D. E. Seborg, T. F. Edgar, D. A. Mellichamp, and F. J. Doyle III, Process Dynamics and Control, 2nd ed., John Wiley \& Sons, 2010.
[2] T. Liu and F. Gao, Industrial Process Identification and Control Design: Step-Test and Relay-Experiment-Based Methods, London, U.K.: Springer-Verlag, 2012.

[3] J. E. Normey-Rico and E. F. Camacho, Control of Dead-Time Processes, London, U.K.: Springer-Verlag, 2007.

[4] J. E. Normey-Rico and E. F. Camacho, "Unified approach for robust dead-time compensator design," J. Process Control, vol. 19, no. 1, pp. 38-47, Jan, 2009.

[5] J. E. Normey-Rico, R. Sartori, M. Veronesi and A. Visioli, "An automatic tuning methodology for a unified dead-time compensator," Control Eng. Pract., vol. 27, pp. 11-22, May. 2014.

[6] P. Albertos and P. Garcia, "Robust control design for long time-delay systems," J. Process Control, vol. 19, no. 10, pp. 1640-1648, Dec. 2009.

[7] P. Garcia and P. Albertos, "Robust tuning of a generalized predictor-based controller for integrating and unstable systems with long time-delay," J.Process Control, vol. 23, no. 8, pp. 1205-1216, Sep. 2013.

[8] V. Lechappe, E. Moulay, F. Plestan, A. Glumineau, and A. Chriette, "New prediction scheme for the control of LTI systems with input delay and unknown disturbances," Automatica, vol. 52, pp. 179-184, Feb. 2015.

[9] Y. L. Chen, T. Liu, P. Garcia, and P. Albertos, "Analytical design of a generalized predictor-based control scheme for low-order integrating and unstable systems with long time delay," IET Control Theorey \& Appl., vol. 10, no. 8, pp. 884-893, May, 2016.

[10] K. H. Ang, G. Chong, Y. Li, "PID control system analysis, design, and technology", IEEE Trans. Control Syst. Technol., vol.13, no.4, pp.559-576, Apr. 2005.

[11] O. J. M. Smith, "Closer control of loops with dead time", Chem. Eng. Prog., vol.53, no.5, pp.217-219, May 1957.

[12] J. E. Normey-Rico and E. F. Camacho, "Dead-time compensator: a survey," Control Eng. Pract., vol. 16, no. 4, pp. 407-428, Apr. 2008.

[13] M. Morari and E. Zafiriou, "Robust Process Control" New Jersey: Prentice Hall, 1989

[14] S. Majhi and D. P. Atherton, "Obtaining controller parameters for a new Smith predictor using autotuning", Automatica, vol.36, no.11, pp. 1651-1658, 2000

[15] W. D. Zhang, D. Y. Gu, W. Wang, and X. Xu. "Quantitative performance design of a modified Smith predictor for unstable processes with time delay," Ind. Eng. Chem. Res., vol. 43. No. 1, pp. 56-62, Jan. 2004.

[16] T. Liu, W. D. Zhang and D. Y. Gu, "Analytical design of two-degree-of-freedom control scheme for open-loop unstable processes with time delay," J. Process Control, vol. 15, no. 5, pp. 559-572, Aug. 2005.

[17] M. R. Mataušek and A. I. Ribić, "Control of stable, integrating and unstable processes by the modified Smith Predictor", J. Process Control, vol.22, no.1, pp.338-343, 2012.

[18] B. C. Torrico, M. U. Cavalcante, A. P. S. Braga, J. E. Normey-Rico, and A. A. M. Albuquerque, "Simple tuning rules for dead-time compensation of stable, integrative, and unstable first-order dead-time processes," Ind. Eng. Chem. Res., vol. 52, no. 33, pp. 11646-11654, Jul. 2013.

[19] P. Zhou, W. Dai, T-Y. Cai, "Multivariable disturbance observer based advanced feedback control design and its application to a grinding circuit”, IEEE Trans. Control Syst. Technol., vol.22, no.4, pp.1474-1485, Apr. 2014.

[20] W-H. Chen, and J. Yang, "Disturbance-observer-based control and related methods-An overview," IEEE Trans. Ind. Electron., vol. 63, no. 2, pp.1083-1095, Feb. 2016

[21] J. Han, "From pid to active disturbance rejection control," IEEE Trans. Ind. Electron., vol. 56, no. 3, pp. 900-906, Feb. 2009.

[22] Z. Gao, "On the centrality of disturbance rejection in automatic control," ISA Trans., vol. 53, , no. 4, pp. 850-857, Jul. 2014.

[23] W. Tan and C. Fu, "Linear active disturbance-rejection control: analysis and tuning via IMC," IEEE Trans. Ind. Electron. vol. 63, no. 4, Apr. 2016.

[24] S. Zhao and Z. Gao, "Modified active disturbance rejection control for time-delay systems," ISA Trans., vol. 53, no. 4, pp. 882-888, Jul. 2014.

[25] Q. Zheng and Z. Gao, "Predictive active disturbance rejection control for processes with time delay," ISA Trans., vol. 53,no. 4, pp. 873-881, Jul. 2014.

[26] R. C. Dorf and R. H. Bishop, Model Control Systems, Menlo Park, CA: Pearson, 1998

[27] A. Seuret, F. Gouaisbaut and E. Fridman, "Stability of discrete-time systems with time-varying delays via a novel summation inequality," IEEE Trans. Autom. Control, vol. 60, no. 10, pp. 2740-2745, Feb. 2015.

[28] K. Gu, V. L. Kharitonov, and J. Chen, Stability of Time-Delay Systems, Boston: Birkhauser, Springer, 2003. 